\title{
High field 3T magnetic resonance imaging: MR-anatomy \& evaluation of traumatic knee injuries
}

\author{
Biswas $\mathrm{A}^{1}$, De $\mathrm{A}^{2}$ \\ ${ }^{1}$ Dr. Anup Biswas, Department of Radiodiagnosis, ${ }^{2}$ Dr. Asim De, Department of Radiodiagnosis, AGMC \& GBP \\ Hospital, Agartala, Tripura, India.
}

Address for Correspondence: Dr. Anup Biswas, Email: docradiology2011@gmail.com

\begin{abstract}
Background: MRI has high applicability to the knees, in comparison with other joints, and it provides excellent diagnostic capacity for evaluating lesions of different types, such as ligament, meniscal, tendon, bone and chondral injuries. MR imaging does not utilize ionizing radiation and therefore it is entirely safe; it is also noninvasive, painless, and allows acquisition of images in multiple planes without repositioning the patient. In addition, MR provides excellent spatial and contrast resolution of both intra- and extra-articular structures. Objective: The purpose of the study was to review the normal MR anatomy of the internal structures of knee joint and to study MRI appearances of various meniscal and cruciate ligament injuries of knee. Results: Knee injuries were more commonly encountered in males as compared to females. ACL was the most commonly injured ligament followed by tears of the menisci. Medial meniscal tears were twice as common as lateral meniscal tears. Vertical and complex tears are slightly more common than horizontal tears, which were predominantly seen in elderly population bearing degenerative changes. PCL tears and tears of the collateral ligaments were rare. Conclusion: Magnetic resonance imaging is a good, accurate and non-invasive modality for the assessment of menisci and ligamenteous injuries. High field 3 T MRI is unique in its ability to evaluate the internal structures of knee with exquisite detail. MRI is highly accurate in the diagnosis of tears of the menisci and cruciate ligaments and thus, it is increasingly used as a first line investigation in patients with soft tissue trauma to knee.
\end{abstract}

Keywords: Magnetic Resonance Imaging (MRI), Medial Meniscus, Lateral Meniscus, Anterior Cruciate Ligament (ACL), Posterior Cruciate Ligament (PCL)

\section{Introduction}

Knee joint is the largest and complex joint of the body and also most frequently injured joint due to the lack of bony support. The stability of the joint is highly dependent on its supporting ligamentous structures, therefore injuries of ligaments and menisci are extremely common [1]. Injury to the knee joint is the significant cause of morbidity in both young and old individuals. An accurate diagnosis regarding the involvement of structures and the extent of injuries is essential for early operative as well as non-operative treatment. This requires an accurate clinical history, a thorough physical examination and complementary diagnostic tools. The most widely used diagnostic modalities to assess the joint injury are arthroscopy and

Manuscript received $26^{\text {th }}$ June 2016

Reviewed: $10^{\text {th }}$ July 2016

Author Corrected: $20^{\text {th }}$ July 2016

Accepted for Publication 31 July 2016
MRI. Arthroscopy is the gold standard to diagnose the intra-articular knee pathology but it is an invasive procedure and can cause complications.

Hence Magnetic resonance imaging (MRI) is increasingly being used for diagnosis over the past decade [2]. MRI has now been accepted as the best imaging modality for non-invasive evaluation of knee injuries [3].

The accuracy, sensitivity and specificity vary in different studies as there are rapid changes in the MRI technology, different imaging sequences, radiologist expertise, diagnostic criteria, location and composition of structure analyzed and the kind of knee injury. As a result of this variability, High filed 3T MRI was 
undertaken to determine the spectrum and common findings encountered in MRI of knee joint injuries [4,5].

\section{Aim}

High Field 3T Magnetic Resonance Imaging: MRanatomy and evaluation of Traumatic Knee Injuries.

\section{Objectives}

To study and evaluate the spectrum of the MRI findings in all the knee joint injuries referred from orthopedic OPD.

\section{Materials \& Methods}

Patients with history of knee joint injury referred from Orthopaedic department for suspected internal derangement of knee joint who underwent MRI were taken for the cross sectional descriptive study. However those patients with ferromagnetic implants, pacemakers, aneurysm clips, patients with knee joint neoplasm, inflammatory and infectious conditions of knee joint were omitted from study. 50 patients were taken by convenient sampling who had undergone MRI between March 2016 to June 2016.

\section{Imaging protocols}

a) Equipment: Siemens Magnetom Skyra 3 Tesla Whole- body MR scanner.

b)Protocol:

- T2 \& STIR sequences in sagittal and coronal planes.

- PD with fat sat weighted sequence in axial, coronal and sagittal planes.

- T1 or 3D DESS sequences wherever indicated.

c) Interpretation of MRI Data

- ACL and PCL : Absent/Present (If present: Partial /Complete) (Site: femoral attachment/ Mid substance/ Tibial attachment)

- Medial and Lateral meniscus tear : Absent/Present (If present: Anterior horn /Body / Posterior horn). (Type:Horizontal/Vertical/Complex)

The associated findings like bone contusion, effusion, loose bodies, parameniscal cysts, baker's cysts and degenerative changes were noted and analyzed. The final diagnosis was made by consultation of two radiologists.

\section{Results}

Knee MRI studies of 50 patients were done fitting into our inclusion criteria. In our study 32 patients were male, and 18 were female; they ranged in age from 12 to 77 years (median, 31 years). The studies included 28 left knees and 22 right knees, one patient had MR imaging of both knees.

High field 3T imaging showed meniscal tears in 32 cases out of 50 cases. Out of the menisci, medial meniscus tear found in 21 cases and was more frequently injured than the lateral meniscus which was torn in 11 cases as shown in Table 1.

Body and posterior horn combined tears were the commonest association followed by isolated posterior horn tears, body and anterior horn tears. Parameniscal cysts were seen in 7 cases associated with meniscal tears.

Table-1: Distribution and frequency of various ligamentous and meniscal tears

\begin{tabular}{|c|c|c|c|}
\hline Sr. No. & Tears & No. of Cases & $\begin{array}{c}\text { Percentage of total patients with knee } \\
\text { injuries }\end{array}$ \\
\hline 1 & Anterior cruciate ligament & 42 & $84 \%$ \\
\hline 2 & Posterior cruciate ligament & 8 & $16 \%$ \\
\hline 3 & Medial meniscus & 21 & $42 \%$ \\
\hline 4 & Lateral meniscus & 11 & $22 \%$ \\
\hline 5 & Lateral collateral ligament & 1 & $2 \%$ \\
\hline 6 & Medial collateral ligament & 4 & $8 \%$ \\
\hline
\end{tabular}

Moreover bucket handle type of meniscal tear was seen in 6 cases, 5 of which were in the medial meniscus. Thus medial meniscus was more commonly involved in bucket handle type tears. Vertical tears, horizontal tears and complex tears were seen in 12, 9 and 11 cases respectively as shown in Table 2. 
Table-2: Various types of Meniscal tears.

\begin{tabular}{|c|c|c|c|}
\hline Sr. No. & Types of Meniscal Tears & No. of cases & Percentage of total meniscal tears \\
\hline 1 & Vertical tears & 12 & $37.5 \%$ \\
\hline 2 & Horizontal tears & 9 & $28.1 \%$ \\
\hline 3 & Bucket handle tears & 6 & $18.8 \%$ \\
\hline 4 & Complex tears & 11 & $34.4 \%$ \\
\hline
\end{tabular}

ACL tears were the commonest finding among the spectrum of knee injuries. ACL tears were seen in 42 cases out of 50 as shown in Table 1. Mid-substance tears were most common, followed by femoral site attachment tears and tibial site attachment tears. Associated underlying bone contusions were also noted in all the cases, bearing tears at the attachment sites. Evidence of joint effusion was also seen in all the cases.

PCL tears were rare and seen in only 8 cases. It was also commonly seen at the mid third associated with buckling of its fibres.

MCL and LCL tears were rare. One case had LCL tear associated with tear of the insertion site of popliteus tendon and semitendinosus. MCL tear was seen in 4 cases and all were found in the mid third of the MCL and associated with meniscocapsular separation. No avulsion of the bony fragments were found.

Degenerative changes were seen in 7 out of 50 cases. Incidental baker's cyst were seen in 3 cases and meniscal fluounce was seen in 1 case.

\section{Discussion}

The study involved review of radiological anatomy of knee and spectrum of findings on MRI which helps in making the diagnosis of various ligamentous and meniscal injuries in cases of knee joint injuries.

\section{MR-anatomy of Important Knee Structures}

Menisci- Menisci are semilunar (C-shaped) fibrocartilaginous structures thick peripherally and thin centrally. Superior surface is concave, inferior surface is flat: with maximum congruency between femur and tibia. Medial meniscus (MM) is larger, wider posterior horn, and more "open" toward intercondylar notch. Lateral meniscus (LM) is smaller, more "closed "toward the notch [6].

Cruciate ligaments- Anterior cruciate ligament $(A C L)$ : running parallel to the roof of the intercondylar notch, from the posteromedial aspect of lateral femoral condyle to the anteromedial tibial plateau. It is intraarticular but extrasynovial and divided into anteromedial bundles and posterolateral. Bundles. Its main function is to restrain anterior tibial translation relative to femur (anteromedial bundle), to restrain rotation in near full extension (posterolateral bundle).

Normal MR imaging shows low T1 and T2 signal, striated appearance lying within the lateral aspect of the intercondylar notch; straight anterior border nearly parallel to the Blumensaat line (roof of intercondylar notch).

Posterior cruciate ligament (PCL): is larger and stronger than ACL. Its main function is to restrain posterior tibial translation relative to femur. It runs from medial side and medial roof of the intercondylar notch to the midline dorsal aspect of the tibial plateau (between meniscal roots). It is intraarticular but extrasynovial and composed of anterolateral and posteromedial bundles. Meniscofemoral ligaments of Humphrey and Wrisberg extend from the posterior horn of the LM to the medial femoral condyle, lying respectively anterior and posterior to the PCL.

Normal MR Imaging shows low T1 and T2 signal, near the midline of the knee, extending from the femoral intercondylar notch to the posterior tibial plateau [7].

Collateral ligaments- Medial collateral ligament (MCL): is extrasynovial structure originating from medial aspect of the distal femur and inserts on the medial aspect of proximal tibia. The fibers intimately linked with joint capsule, and the MM is directly attached to it. 
Lateral Collateral Ligament (LCL): is made up of many structures, only three are analysed by MRI: (posterior to anterior): biceps femoris tendon, fibulocollateral ligament and iliotibial band. The most posterior two insert on proximal fibula whereas the iliotibial band inserts on anterior tibia.

\section{Spectrum of imaging findings helping to make the diagnosis of various ligamentous and meniscal injuries}

The majority of knee joint injuries resulted from the direct trauma to the knee joint or was caused by torsional or angulatory forces. These injuries vary in severity from simple ligamentous strain to complex injuries involving ligamentous disruption with meniscal damage and associated fracture $[8,9]$.

Diagnosis of ACL Tears: The primary sign of ACL tear is fibre discontinuity which was best observed in the oblique sagittal plane proving most helpful in diagnosing ACL tears. The empty notch sign on coronal imaging was a frequent finding in complete ACL tear [10]. Bone contusion was very common in ACL tears (Figure 1). [9].

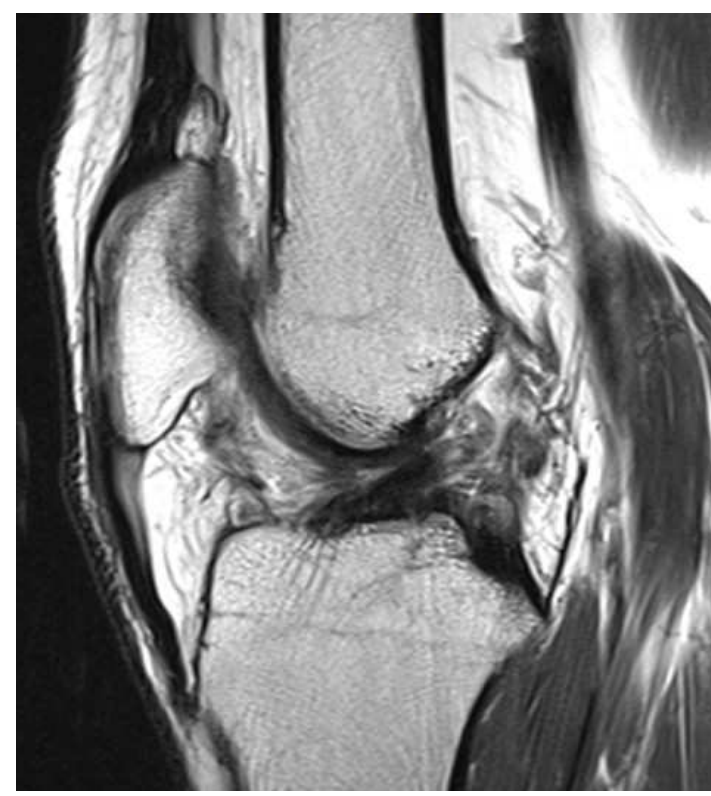

Fig.-1: Complete ACL tear

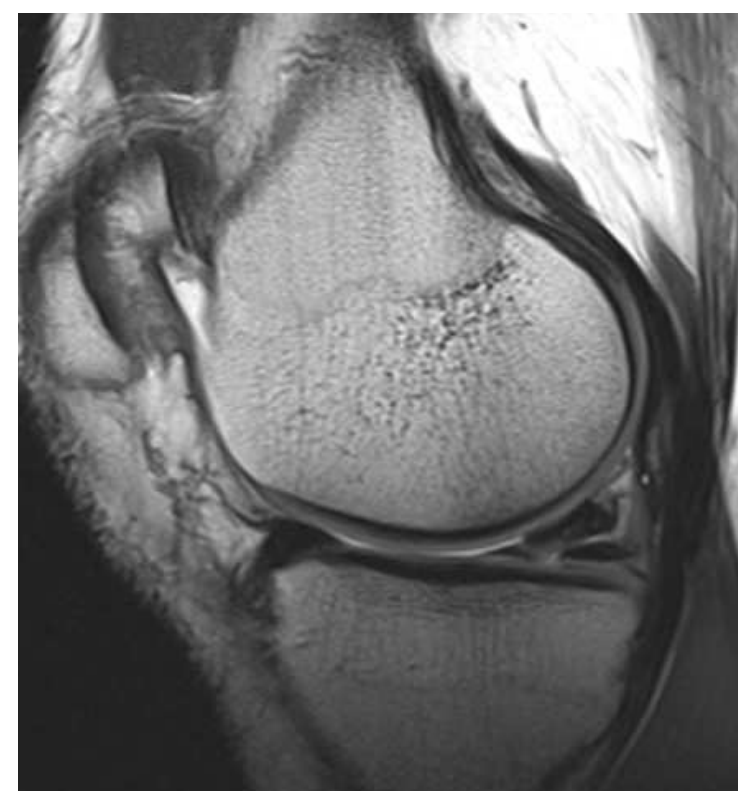

Fig.-2: Complex medial meniscal tear

In acute or sub-acute injury, thickening and oedema of the ACL was found characterized by increased signal intensity on T2 or PD with fat sat weighted sequences. In chronic cases the fibre were completely or partially thinned out.

Oblique coronal and sagittal views parallel to the ACL have been advocated and found to be effective in improving visualization of the ACL. However the sensitivity and specificity of MRI in the diagnosis of partial tears was poor.

Diagnosis of PCL Tears: Being a much stronger ligament complete disruption of PCL was less common and was usually associated with severe knee trauma and injuries to posterior lateral corner structures. Partial or intra substance tears are much more common and manifested as T2 or STIR intra substance hyperintensity with often laxity and thickening of the ligament [10].

Diagnosis of Posteromedial corner (PMC) Injury: Hyperextension and valgus is usually the mechanism responsible for injury to the posteromedial corner. The PMC injuries can be divided into injuries to the ligaments (MCL and POL injuries), Menisco-capsular injury and Musculotendinous injury to the semimembranosus insertion.

Ligament injury: Three grades of MCL injury have been described- Grade I injury is a sprain with high signal in the soft tissues medial to the MCL, Grade 2, a severe sprain or partial tear, with high signal in the soft tissues medial to the MCL with partial disruption of the MCL. Grade 3, complete tear, shows disruption of the MCL and disruption of medial patellar retinaculum. (Figure 4). 


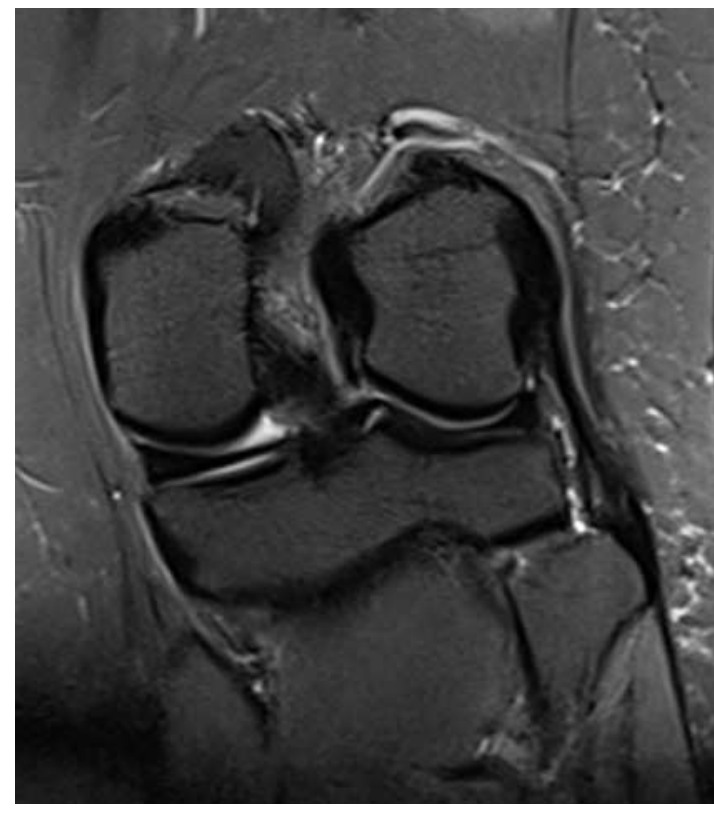

Fig.-3: Partial LCL tear

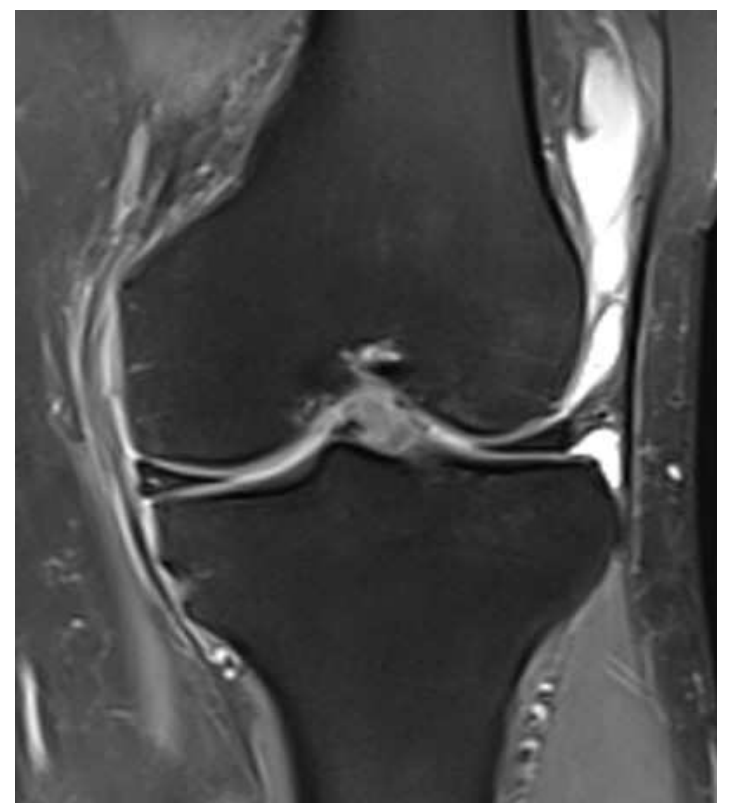

Fig.-4: Partial MCL tear, grade I MM tear and periarticular effusion

Menisco-capsular injury: MRI readily demonstrated injury of the femoral and tibial meniscal attachments as disruption and thickening of the menisco tibial and menisco femoral ligaments with or without associated marrow edema at the ligament insertion.

Musculotendinous injury: Damage to the semimembranosus insertion is common in posteromedial knee injuries. Injury to the semimembranosus tendinous expansions may include avulsion fracture of the tibial insertion, complete or partial tears of the tendon and chronic insertional tendinosis. Capsular injury manifests as thickening and hyperintensity of the posterior capsule at the level of the medial femoral condyle [11].

Diagnosis of Posterolateral corner (PLC) Injury: The stability of the posterolateral corner of the knee is provided by capsular and noncapsular structures that function as static and dynamic stabilizers. They include Illiotibial band, Joint capsule, Biceps femoris tendon, Fibulocollateral ligament, Popliteus muscle and tendon, Popliteofibular and Arcuate ligaments.

There is no specific MRI criteria for defining posterolateral corner injury, however injuries to the posterolateral ligamentous structures can be classified as grade I, II, or III sprains, corresponding to minimal, partial, or complete tearing, respectively. Injury to two or more major stabilizing structures especially the popliteus myotendinous unit, fibular collateral ligament and posterior lateral joint capsule should be considered as posterolateral corner injury. Grade 3 injuries are often associated with disruption of the cruciate ligaments.

Injuries to the fibular collateral ligament are better appreciated in coronal fat suppressed proton density (PD) or coronal fat suppressed $\mathrm{T} 2 \mathrm{~W}$ images.

Injuries range from partial intra substance tear to complete disruption and avulsion from bony attachment from fibular head, periligamentous soft tissue edema is often present. (Figure 3).

The bony injuries associated with PLC injuries include marrow contusions involving the anteromedial femoral condyle, anterior medial tibial margin fracture and "Arcuate" fracture which represents avulsion fracture of the fibular styloid process $[12,13]$.

Diagnosis of Meniscal Tears: A meniscal tear only has significance when hyperintense signal clearly disrupts at least an articular surface of the meniscus. Description of meniscal tears should include: 
- Location: on posterior horn, body or anterior horn

- Involving: of peripheral third of the meniscus( vascularized red zone) or inner two thirds or both

- Extent of the tear: which meniscal surface-length

- Type of meniscal tears: horizontal (oblique), vertical (longitudinal, radial or parrot-beak) or complex.

Different types of meniscal tears: Meniscal tears can be broadly divided into horizontal, vertical circumferential and vertical radial types. Combination of the main types are not unusual. Usually vertical tears are traumatic and horizontal tears are degenerative.

1. Horizontal or oblique tears (synonymous): Tears are parallel to tibial plateau, dividing meniscus into upper and lower segments.

2. Vertical longitudinal tear: perpendicular to tibial plateau.

3. Bucket-handle tear: $10 \%$ of meniscal tears and most common lesion in the locked knee: the free inner meniscal segment "flips" usually into the intercondylar notch; involves more frequently the MM.

- Absent bow tie sign: only one body segment on sagittal images instead of two normal images seen through the meniscus, very sensitive sign in all cases.

- Double PCL sign: displaced fragment usually seen on intercondylar notch or in front of the PCL.

- Anterior flipped meniscus sign: when the displaced fragment flips over the anterior horn of the meniscus.

4. Vertical radial tears or free edge tears: occur on a plane perpendicular to the long axis of the meniscus and perpendicular to the tibial plateau; usually symptomatic.

- Absent bow tie sign: also positive, but the second body segment has only a small gap rather than the large space seen in bucket-handle fractures, and no displaced fragment is seen.

- Ghost meniscus sign: is seen when the tear has completely traversed the meniscus with an MRI slice parallel to the tear: intermediate or grey signal (partial volume of the adjacent meniscal tissues) instead of normal meniscus; meniscus often extrudes off of the joint.

- Cleft sign: seen when the MRI slice is perpendicular to the tear while the radial tear takes a truncated triangle appearance when the MRI slice is parallel to the same tear with a cleft.

5. Complex tears: tears having either two or more tear configurations or are not categorized easily into a certain type of tear. (Figure 2).

\section{Particular cases:}

- Vertical parrot-beak tears are radial at the inner meniscal edge and longitudinal more peripherally within the meniscus-Detection by MR imaging difficult.

- Flap tears are displaced fragment from the superior or inferior surface of the meniscus. The displaced portion is usually easy to see on MRI $[13,14,15]$.

\section{Conclusion}

MRI has proved reliable \& safe. Since its introduction for clinical use in the mid-1980s, the MRI has emerged as a primary tool, to guide the management of knee injuries. With the development of new sequences, improved signal to noise ratio, high resolution, reduced artifacts and shorter imaging times the, role of MRI in the diagnosis of patients with knee complaints has improved drastically. With the advent of High Field 3 Tesla MRI scanners, which provides excellent details with high accuracy, MRI has become the prime investigation of choice for patients coming with Knee injuries.

Funding: Nil, Conflict of interest: None initiated, Permission from IRB: Yes

\section{References}

1. Li DK, Adams ME, McConkey JP. Magnetic resonance imaging of the ligaments and menisci of the knee. Radiol Clin North Am. 1986 Jun;24(2):209-27. 
2. Mahmoud Karimi-Mobarake, Hamid BaraniBaravati. The Accuracy of Magnetic Imaging Compared with Arthroscopic Finding in Intra-articular Traumatic Knee Injury. Journal Applied Sciences 2005; 5(4): 686-688.

3. Winters K, Tregonning R. Reliability of magnetic resonance imaging of the traumatic knee as determined by arthroscopy. N Z Med J. 2005 Feb 11;118 (1209):U1301.

4. Bryan S, Weatherburn G, Bungay H, Hatrick C, Salas C, Parry D, Field S, Heatley F. The cost-effectiveness of magnetic resonance imaging for investigation of the knee joint. Health Technol Assess. 2001;5(27):1-95.

5. Langer JE, Meyer SJ, Dalinka MK. Imaging of the knee. Radiol Clin North Am. 1990 Sep;28(5):975-90.

6. Raunest J, Hotzinger H, Burrig KF. Magnetic resonance imaging (MRI) and arthroscopy in the detection of meniscal degenerations correlation of arthroscopy and MRI with histology findings. Arthroscopy 1994; 10(6):634-640.

7. Lee JK, Yao L, Phelps CT, Wirth CR, Czajka J, Lozman J. Anterior cruciate ligament tears: MR imaging compared with arthroscopy and clinical tests. Radiology. 1988 Mar;166(3):861-4.

8.Mackenzie R, Dixon AK, Keene GS, Hollingworth W, Lomas DJ, Villar RN. Magnetic resonance imaging of the knee: assessment of effectiveness. Clin Radiol. 1996 Apr;51(4):245-50.

9. Munk B, Madsen F, Lundorf E, Staunstrup H, Schmidt SA, Bolvig $\mathrm{L}$ et al. Clinical magnetic resonance imaging and arthroscopic findings in knees: a comparative prospective study of meniscus anterior cruciate ligament and cartilage lesions. Arthroscopy, 1998; 14(2):171-175.

10. MR imaging of the posterior cruciate ligament: normal, abnormal, and associated injury patterns. Sonin AH, Fitzgerald SW, Hoff FL, Friedman H, Bresler ME. Radiographics. 1995 May;15(3):551-61.

11. Bencardino JT, Rosenberg ZS, Brown RR, Hassankhani A, Lustrin ES, Beltran J. Traumatic musculotendinous injuries of the knee: diagnosis with MR imaging. Radiographics. 2000 Oct; 20 Spec No:S103-20.

12. JP Singh, L Garg, R Shrimali, V Setia, V Gupta. MR Imaging of knee with Arthroscopic Correlation in Twisting Injuries. Ind J Radio 2004 14; 1:33-40.

13. Ruth Crawford, Gayle Walley, Stephen Bridgman, Nicola Maffulli. Magnetic resonance imaging versus arthroscopy in the diagnosis of knee pathology, concentrating on meniscal lesions and ACL tears: a systematic review. British Medical Bulletin 2007; 84: 5-23.

14. Mesgarzadeh M, Moyer R, Leder DS, Revesz G, Russoniello A, Bonakdarpour A, Tehranzadeh J, Guttmann D. MR imaging of the knee: expanded classification and pitfalls to interpretation of meniscal tears. Radiographics. 1993 May;13(3):489-500.

15. Fox MG. MR imaging of the meniscus: review, current trends, and clinical implications. Magn Reson Imaging Clin N Am. 2007 Feb;15(1):103-23.

\section{How to cite this article?}

Biswas A, De A. High field 3T magnetic resonance imaging: MR-anatomy \& evaluation of traumatic knee injuries. Int J Med Res Rev 2016;4(8):1323-1329.doi:10.17511/ijmrr.2016.i08.08. 